\title{
A Study on Hand Contamination and Hand Washing Practices among Medical Students
}

\author{
Watutantrige Ranjit De Alwis, Premalatha Pakirisamy, \\ Lum Wai San, and Evelyn Chen Xiaofen
}

Division of Community Medicine, International Medical University, 126, Jalan Jalil Perkasa 19, Bukit Jalil, 57000 Kuala Lumpur, Malaysia

Correspondence should be addressed to Premalatha Pakirisamy, premalatha_pakirisamy@imu.edu.my

Received 6 January 2012; Accepted 6 February 2012

Academic Editors: E. C. Alexopoulos and K. McLeroy

Copyright (C 2012 Watutantrige Ranjit De Alwis et al. This is an open access article distributed under the Creative Commons Attribution License, which permits unrestricted use, distribution, and reproduction in any medium, provided the original work is properly cited.

\begin{abstract}
Harmful microorganisms can be transferred to hands from contaminated surfaces people come into contact in daily life. Contaminated hands can transmit disease to one self as well as to others. A study was done to determine the extent to which hand hygiene practices and toilet door knobs contribute to the bacterial load of hands of toilet users in a medical school. Swabs were taken from a randomly selected sample of 60 medical students for bacterial count from both hands before and after toilet use and from door knobs of six toilets. Only $40(66.7 \%)$ claimed they washed hands with soap. Significantly more females (83\%) used soap to wash hands compared to males (50\%). Bacterial load in the hands of both males and females showed an increase after toilet use. The increase was significant among male students. The dominant hand had a significantly higher bacterial load than the other. The mean bacterial load of male toilet door knobs $\left(12 \mathrm{CFU} / \mathrm{cm}^{2}\right)$ were significantly higher than of female toilet door knobs $\left(2.5 \mathrm{CFU} / \mathrm{cm}^{2}\right)(P<0.05)$. Staphylococcus aureus was isolated from the hands of 21 students. Toilets and washrooms should be designed so as to eliminate the sources of contamination of the hands.
\end{abstract}

\section{Introduction}

Infection transmission through contaminated hands of health care workers $(\mathrm{HCW})$ is a common pattern seeing in most healthcare settings $[1,2]$. Failure to perform appropriate hand hygiene practices is a leading cause of health careassociated infections (HCAI) and the spread of multiresistant organisms and has been recognised as a significant contributor to outbreaks of infectious diseases by the World Health Organisation (WHO) [3]. WHO recognises that washing hands of HCWs with soap can prevent infection in patients and is the most effective and inexpensive way to prevent transmission. The United Nations General Assembly designated 2008 as the International Year of Sanitation [4]. To reinforce the United Nation's call for improved hygiene practices, the Global Public-Private Partnership for Hand washing declared 15 October 2008 as the first Global Hand washing Day with the objective of promoting a global culture of hand-washing with soap [5]. This campaign was initiated mainly to reduce mortality rates related to diarrhoeal diseases by introducing simple behavioural changes. As part of a major global effort to improve hand hygiene in health care, the WHO in 2009 launched a global campaign named "SAVE LIVES: Clean Your Hands" [6]. Interestingly a directive for healthcare workers to practise hand hygiene was made as far back as 1847 by Ignas Semmelweis in Vienna [7]. In spite of the wisdom of Semmelweis, poor hand-washing compliance rates continue to exist in the general population [8] and in health care settings [9-11].

A range of strategies for the promotion and improvement of hand hygiene is recommended in the hand hygiene guidelines published by the Centre for Disease Control (CDC) Atlanta $[12,13]$ and the WHO [3]. In spite of institutional policies and protocol on hand hygiene, observational studies show poor hand hygiene compliance among HCWs [9-13]. A notable risk factor consistently associated with poor adherence to recommended hand-hygiene practices observed in 
healthcare was doctors rather than nurses $[13,14]$. Hence it is crucial that medical schools producing future healthcare workers train with a view towards changing the behaviour of medical students on hand hygiene practice. Hand washing before attending to patients as well as after contact with any possible source of contamination such as the toilet is an essential practice that students should learn to adopt. Two distinct behavioural practices concerning hand hygiene are described: inherent hand hygiene which drives the majority of community hand washing behaviour such as washing when the hands are visibly dirty or after eating or after toilet use and elective hand hygiene when contacts are not perceived to pose a threat and does not trigger an intrinsic response with an immediate desire to wash hands. Situations requiring such elective hand washing are frequently encountered in healthcare by protocols [15]. Although hand hygiene is of high importance, the emphasis given in the curriculum for hand hygiene varies from institute to institute. Even though most medical schools do have a module on personal and professional development where emphasis is laid on communication skills and professionalism, hardly any focus is given to personal hygiene or social etiquette. Whether there is actual awareness of and compliance with hand hygiene among students in a medical school is not known. An observational study conducted to evaluate hygiene habits of students at a university in Alabama found that female students had a higher tendency to wash their hands more often than male students, and science major students had the highest compliance compared with nonscience students [16]. Another observational study of hand-washing practices among female students in a liberal arts college in the mid-Atlantic region found that not all the students washed their hands after toilet use. While $63 \%$ washed their hands, $38 \%$ used soap, but only $2 \%$ washed for over 10 seconds [17]. Through cultivation of good hand hygiene habits in medical schools, students will eventually practise these good habits later in their working life. While considerable attention is given to hand hygiene practices, little efforts are taken to eliminate the sources and risk of hand contamination. A series of measures were taken in this medical school to promote hand hygiene after toilet use in addition to skills in hand washing taught in the curriculum. In all the toilets and in other strategic points, illustrated WHO recommended steps on hand-washing were placed. A banner with the caption "Have you washed your hands?" was fixed on the inner side of exit doors in all the toilets to remind students to wash their hands before leaving the toilet. Liquid soap dispensers were installed with hand driers in all the toilets. Alcohol-based hand rub dispensers were installed in many strategic points.

This study was done to determine the extent to which toilet door knobs are a source of the hands of users being contaminated and also to determine hand hygiene practices among medical students in a medical school. The findings of this study will help to eliminate sources of hand contamination through better design of toilets and to promote the practice of keeping hands clean among medical students, who as future doctors will contribute to reducing HCAI if they develop this habit.

\section{Materials and Methods}

Considering the resources available, we decided to randomly select a sample of 60 students ( 30 males and 30 females) from the semester four class of 200 medical students consisting of 106 females and 94 males for this study. Every third student entering the toilet during the fifteen-minute break between lectures in the morning session and afternoon session on five consecutive working days was selected. The number selected per session was restricted to six (three males and three females). After obtaining consent from the selected students, swabs were taken from both right and left hands of students before they entered the toilet and immediately after they exited the toilet. Students were not told before they entered the toilet that a swab would be taken after toilet use. Hence consent was requested again for swabbing their hands. Students were also requested to answer several questions regarding their hand-washing practice after exiting the toilet. Students who used the toilet for micturition only were included in this study. Those using the toilet for defaecation were excluded. Each toilet unit had three cubicles and two wash basins. The male toilet in addition had a urinal. The toilet unit had an exit door, which had a door knob to turn to open. Swabs were taken from the inside knob of the exit door of six toilet units (three male and three female) in the morning and evening on Monday, Wednesday, and Friday during the survey.

Sterile cotton swabs dampened in $0.85 \%$ saline were used to take samples from the palm, fingers, and between fingers. Sterile cotton swabs premoistened with $0.85 \%$ saline were rotated around the circumference of the door knob to obtain samples from the selected toilets. The cotton swabs were transported in $50 \mathrm{~mL}$ sterile saline and processed in the laboratory immediately. $0.5 \mathrm{~mL}$ of each sample was then inoculated in Tryptic soy agar plates (Merck). Plates were incubated at $37^{\circ} \mathrm{C}$ under aerobic conditions, and colony-forming units (CFUs) were counted after 24 to $48 \mathrm{~h}$; the colony forming units ranged between 100 and 250. The mean of colony counts from the palm, finger, and between fingers was taken as the bacterial load of that hand. The representative colonies were Gram stained. Bacterial identification was performed by means of sequencing the 16S rDNA region, and the sequences were analysed against a bacterial DNA genome database using the National Center for Biotechnology Information's (NCBI) Basic Local Alignment Search Tool (BLAST). Bacteria DNA was extracted using a DNA extraction kit (Thermo Scientific).

Bacterial counts were expressed as the number of CFUs per hand. Mean CFU was calculated from triplicate samples. Bacterial load on hands was compared using the $\log _{10}$ transformed bacterial count. Mann-Whitney $U$ test and Wilcoxon Signed Ranks test were done to test the significance of the difference in mean CFU counts between right and left hands and between before and after toilet use. The choice of this statistical test is appropriate for studies with a small sample size and when the variables of interest do not show normal distribution. The level of significance was at $P<0.05$. The bacterial load of door knobs was expressed as CFU per $\mathrm{cm}^{2}$ by dividing the CFU count by the square area of the door knob. 
The study protocol was approved by the University's joint committee of research and ethics.

\section{Results and Discussion}

All 60 students claimed to have washed their hands after using the toilet. When asked about soap use, 40 (66.7\%) subjects claimed to have washed their hands with soap while the rest claimed to have washed their hands with water only. Among the female students $83 \%$ used soap, while only $50 \%$ of male students said they used soap. Hand-washing after toilet use was reported by all subjects with or without soap. However, this may be an overestimate as it is possible that the responses given were intended to be socially acceptable. Although students were not told that samples would be taken after their use of the toilet, they may have known that swabs would be taken from their hands after toilet use. However, $33 \%$ did not use soap on their own admission in spite of knowing that they will be sampled. The duration of washing and the technique of washing were not taken into account and both are crucial in the disinfecting of all areas of the hand. Female students expressed better hand-hygiene practices than did the male students. These results were coherent with previous observational studies. Females were reported to wash hands more often as well as more thoroughly [16-20] and had less bacterial load on their hands than the males [21, $22]$. The reason for this consistent variation between genders remains debatable although one study on female hand washing practices showed that females tend to improve hand hygiene habits in the presence of other toilet users in the sink area [17].

The CFU count of hands obtained before toilet use showed no significant difference between the right and left hand in both males and females (Mann-Whitney $U$ test, right hand $P=0.097$, left hand $P=0.096)$, Table 1 . Swabs were taken from the palm, fingers, and between fingers. Hence it may not represent the total bacterial load of the hand. While there are many methods of hand sampling described in the literature, this method of taking hand swabs was used in a study of hand flora in Turkish nurses [23]. The mean CFU count of both right and left hands of the students was significantly higher after toilet use compared to the baseline bacterial load on hands before toilet use (Wilcoxon Signed Ranks test, right hand $P=0.001$, left hand $P=0.003$ ). However, when the bacterial load on hands of male and female students were looked at separately, there was a significant increase in bacterial load on both hands after toilet use among the male students (Wilcoxon Signed Ranks test, right hand $P<0.001$, left hand $P<0.001$ ), whereas no significant increase was seen among the female students. (Wilcoxon Signed Ranks test, right hand $P=0.405$, left hand $P=0.456$ ). This was expected because more females used soap to wash hands as compared to males. Another factor that could be implicated is the difference in natural micturition habits between males and females. Males would have more direct contact with the genitalia, which could be a source of contamination. Apart from this, the position of males facing the urinal bowl would also potentially expose their hands to a degree of aerosols splashing from the urinary bowls.
TABLE 1: Bacterial load of hands of subjects before and after toilet use by gender.

\begin{tabular}{lcccc}
\hline & Hand & \multicolumn{2}{c}{$\log _{10}$ mean CFU \pm SD } & \\
& & Before & After & $P$ Value \\
\hline \multirow{2}{*}{ Overall (N 60) } & Right & $3.22 \pm 0.70$ & $3.55 \pm 0.66$ & 0.001 \\
& Left & $3.17 \pm 0.87$ & $3.48 \pm 0.79$ & 0.003 \\
Males (N 30) & Right & $3.36 \pm 0.64$ & $3.91 \pm 0.56$ & 0.001 \\
& Left & $3.39 \pm 0.59$ & $3.78 \pm 0.54$ & 0.001 \\
Female $(N$ 30) & Right & $3.09 \pm 0.75$ & $3.20 \pm 0.56$ & 0.405 \\
& Left & $2.94 \pm 1.05$ & $3.19 \pm 0.89$ & 0.456 \\
\hline
\end{tabular}

TABLE 2: Bacterial load of hands before and after toilet use according to washing practices.

\begin{tabular}{lcccc}
\hline & \multirow{2}{*}{ Hand } & \multicolumn{2}{c}{$\log _{10}$ mean CFU \pm SD } & \multirow{2}{*}{ P value } \\
& & Before & After & \\
\hline \multirow{2}{*}{ With soap $(n=40)$} & Right & $3.17 \pm 0.71$ & $3.41 \pm 0.61$ & 0.033 \\
& Left & $3.16 \pm 0.81$ & $3.32 \pm 0.82$ & 0.182 \\
Without soap $(n=20)$ & Right & $3.32 \pm 0.68$ & $3.83 \pm 0.68$ & 0.007 \\
& Left & $3.18 \pm 1.01$ & $3.81 \pm 0.61$ & 0.003 \\
\hline
\end{tabular}

The baseline bacterial load on the hands of those who washed with soap and those who did not use soap was not significantly different. Students who did not wash their hands with soap showed a significant increase in bacterial load after toilet use (Wilcoxon Signed Ranks test, right hand $P=0.007$, left hand $P=0.003$ ). Interestingly those who washed with soap also showed an increase in bacterial load after toilet use but this increase was statistically significant only on the right hand. Bacterial load on the hands of those who washed their hands with soap had a significantly lower count after toilet use than those who washed with water only (MannWhitney $U$ test, right hand $P=0.023$, left hand $P=0.014$ ). Table 2. Although we are not certain of the duration and the technique of those who used soap to wash hands, it is quite possible that their hands may have got contaminated from touching surfaces such as the tap and the door knob inside the toilet after they had washed their hands. The significant increase of bacterial load on the right hand of those who washed their hands strengthens the suspicion that contamination might have occurred after washing hands from touching toilet door knobs, as the right hand being the dominant hand would have being used to open the toilet door to exit. Previous studies found that uneven surfaces like the door knobs and taps were the least cleaned areas, and therefore they contained the highest bacterial load in a toilet $[24,25]$.

The male toilet door knobs (mean $12.5 \mathrm{CFU} / \mathrm{cm}^{2}$, range 0.74-21.66) had a statistically significant $(Z=-3.467, P<$ 0.05 ) higher colony count than the female toilet door knobs (mean $3.04 \mathrm{CFU} / \mathrm{cm}^{2}$, range $0.18-21.3$ ). This difference again could be due to the gender difference in hand washing practices and hand bacterial load seen in our study resulting in less contamination of door knobs by females. There was no significant difference in the colony counts between samples taken in the morning and evening. $(Z=-0.382, P>0.05)$, 
or between the samples taken during different days of the week $(X 2=0.142, P>0.05)$ probably due to the cleaning of the toilets three times a day.

A total of 38 unique bacteria colonies were isolated based on colony morphology from the hands of subjects after toilet use. There were a total of 27 Gram positive isolates and a total of 11 Gram negative isolates. Of the 27 Gram positive isolates, 21 were cocci and six were bacilli. Of the $11 \mathrm{Gram}$ negative isolates, three were cocci and eight were bacilli. 18 of the total 38 isolated bacteria were successfully amplified using the PCR technique, and only 10 different species were identified. Successful PCR amplification was confirmed through gel electrophoresis, which yielded bands at specific size for Staphylococcus aureus, Staphylococcus haemolyticus Staphylococcus capitis, Staphylococcus hominis, Staphylococcus epidermidis, Kocuria rhizophila, and Arthrobacter aurescens. Staphylococcus aureus was isolated from the hands of 21 students ( 18 males and 3 females) after toilet use of which 15 belonged to the group that did not use soap to wash hands. Before toilet use, Staphylococcus aureus was isolated from the hands of only three students. The bacteria identified from the hands of the students belong to the normal flora of the hand and pose no significant health hazard to healthy individuals. However, the normal flora of the hands of HCWs could be pathogenic especially in immunocompromised patients [26]. The isolation of Staphylococcus aureus from the hands of 21 students is significant. Staphylococcus aureus is one of the most common causes of both community and hospital acquired infections [27]. Staphylococcus aureus is known to be the causative pathogen in a range of diseases: impetigo [28], folliculitis [29], septic arthritis [30], osteomyelitis [31], septicaemia [32], pneumonia [33], and meningitis [34]. Students hands may have got contaminated with staphylococcous aureus either by contact with the genital area or by contact with contaminated toilet surfaces.

Through DNA sequencing Staphylococcus, aureus, Staphylococcus haemolyticus, Staphylococcus haemolyticus, Staphylococcus capitis, Staphylococcus hominis, Staphylococcus epidermidis, Kocuria rhizophilla, and Arthrobacter aurescens were identified from the door knob samples based on high similarity in the available sequential database.

\section{Conclusions}

In spite of the training and performance under supervision during exams and the hand hygiene promotion activity, this study revealed that the students did not have a culture of good hand hygiene practice.

Our study showed that female medical students displayed better hand hygiene practices than male medical students.

It is very likely that the door knobs were contaminated by the hands of students who did not use soap to wash their hands after micturition.

Toilet door knobs were a source of hand contamination even among those who washed hands with soap after the use of the toilet. Hence public toilets should be designed so that while privacy is maintained, the user will not have doors that he or she might have to touch on the way out of the washing area.

\section{Acknowledgments}

The auhors thank Professor Kumudu Wijewardene and Ms. Crescentia Morais for reviewing paper and providing suggestions for improvements. They thank the International Medical University for providing funds for this paper.

\section{References}

[1] D. Pittet, B. Allegranzi, H. Sax et al., "Evidence-based model for hand transmission during patient care and the role of improved practices," The Lancet Infectious Diseases, vol. 6, no. 10, pp. 641-652, 2006.

[2] J. M. Boyce, G. Potter-Bynoe, S. M. Opal, L. Dziobek, and A. A. Medeiros, "A common-source outbreak of Staphylococcus epidermidis infections among patients undergoing cardiac surgery," Journal of Infectious Diseases, vol. 161, no. 3, pp. 493499, 1990.

[3] World Health Organization, WHO Guidelines on Hand Hygiene in Healthcare, WHO, Geneva, Switzerland, 2009.

[4] United Nation, International Year of Sanitation, http://esa .un.org/iys/background.shtml.

[5] Global Public-Private Partnership for Handwashing: Global handwashing day 15 October, http://www.globalhandwashingday.org/About.asp.

[6] World Health Organisation, "Clean care is safe care," http:// www.who.int/gpsc/en/.

[7] W. R. Jarvis, "Handwashing-the Semmelweis lesson forgotten?” The Lancet, vol. 344, no. 8933, pp. 1311-1312, 1994.

[8] V. Curtis and S. Cairncross, "Effect of washing hands with soap on diarrhoea risk in the community: a systematic review," The Lancet Infectious Diseases, vol. 3, no. 5, pp. 275-281, 2003.

[9] D. Pittet, "Compliance with hand disinfection and its impact on hospital-acquired infections," Journal of Hospital Infection, vol. 48, supplement A, pp. S40-S46, 2001.

[10] R. K. Albert and F. Condie, "Hand-washing patterns in medical intensive-care units," The New England Journal of Medicine, vol. 304, no. 24, pp. 1465-1466, 1981.

[11] M. Graham, "Frequency and duration of handwashing in an intensive care unit," American Journal of Infection Control, vol. 18, no. 2, pp. 77-81, 1990.

[12] J. S. Garner and M. S. Favero, "CDC Guideline for handwashing and hospital environmental control, 1985," Infection Control, vol. 7, no. 4, pp. 231-243, 1986.

[13] J. M. Boyce and D. Pittet, "Guideline for hand hygiene in healthcare settings: recommendations of the healthcare infection control practices advisory committee," Infection Control and Hospital Epidemiology, vol. 23, supplement 12, pp. 3-39, 2002.

[14] D. Pittet, P. Mourouga, and T. V. Perneger, "Compliance with handwashing in a teaching hospital," Annals of Internal Medicine, vol. 130, no. 2, pp. 126-130, 1999.

[15] M. Whitby, M. L. McLaws, and M. W. Ross, "Why healthcare workers don't wash their hands: a behavioral explanation," Infection Control and Hospital Epidemiology, vol. 27, no. 5, pp. 484-492, 2006.

[16] J. K. Taylor, R. Basco, A. Zaied, and C. Ward, "Hand hygiene knowledge of college students," Clinical Laboratory Science, vol. 23, no. 2, pp. 89-93, 2010.

[17] D. Drankiewicz and L. Dundes, "Handwashing among female college students," American Journal of Infection Control, vol. 31, no. 2, pp. 67-71, 2003.

[18] A. K. Halder, C. Tronchet, S. Akhter, A. Bhuiya, R. Johnston, and S. P. Luby, "Observed hand cleanliness and other measures 
of handwashing behavior in rural Bangladesh," BMC Public Health, vol. 10, article 545, 2010.

[19] M. E. Guinan, M. McGuckin-Guinan, and A. Sevareid, "Who washes hands after using the bathroom?" American Journal of Infection Control, vol. 25, no. 5, pp. 424-425, 1997.

[20] J. H. Park, H. K. Cheong, D. Y. Son, S. U. Kim, and C. M. $\mathrm{Ha}$, "Perceptions and behaviors related to hand hygiene for the prevention of $\mathrm{H} 1 \mathrm{~N} 1$ influenza transmission among Korean university students during the peak pandemic period," $B M C$ Infectious Diseases, vol. 10, article 222, 2010.

[21] A. J. Pickering, A. B. Boehm, M. Mwanjali, and J. Davis, "Efficacy of waterless hand hygiene compared with handwashing with soap: a field study in Dar es Salaam, Tanzania," The American Journal of Tropical Medicine and Hygiene, vol. 82, no. 2, pp. 270-278, 2010.

[22] A. M. Snelling, T. Saville, D. Stevens, and C. B. Beggs, "Comparative evaluation of the hygienic efficacy of an ultra-rapid hand dryer vs conventional warm air hand dryers," Journal of Applied Microbiology, vol. 110, no. 1, pp. 19-26, 2011.

[23] R. B. Akpinar, A. Celebioglu, H. Uslu, and M. Hamidullah Uyanik, "An evaluation of the hand and nasal flora of Turkish nursing students after clinical practice," Journal of Clinical Nursing, vol. 18, no. 3, pp. 426-430, 2009.

[24] P. C. Carling, M. F. Parry, and S. M. Von Beheren, "Identifying opportunities to enhance environmental cleaning in 23 acute care hospitals," Infection Control and Hospital Epidemiology, vol. 29, no. 1, pp. 1-7, 2008.

[25] P. C. Carling, S. Von Beheren, P. Kim, and C. Woods, "Intensive care unit environmental cleaning: an evaluation in sixteen hospitals using a novel assessment tool," Journal of Hospital Infection, vol. 68, no. 1, pp. 39-44, 2008.

[26] M. Wilson, Microbial Inhabitants of Humans: Their Ecology and Role in Health and Diseases, Cambridge University Press, Cambridge, Mass, USA, 1st edition, 2005.

[27] A. I. Hidron, J. R. Edwards, J. Patel et al., "Antimicrobial-resistant pathogens associated with healthcare-associated infections: annual summary of data reported to the National Healthcare Safety Network at the Centers for Disease Control and Prevention, 2006-2007," Infection Control and Hospital Epidemiology, vol. 29, no. 11, pp. 996-1011, 2008.

[28] R. Dagan, "Staphylococcus aureus in Impetigo," American Journal of Diseases of Children, vol. 145, no. 11, p. 1223, 1991.

[29] M. J. Sladden and G. A. Johnston, "More common skin infections in children," British Medical Journal, vol. 330, no. 7501, pp. 1194-1198, 2005.

[30] D. L. Goldenberg and J. I. Reed, "Bacterial arthritis," The New England Journal of Medicine, vol. 312, no. 12, pp. 764-771, 1985.

[31] F. A. Waldvogel and H. Vasey, "Osteomyelitis: the past decade," The New England Journal of Medicine, vol. 303, no. 7, pp. 360 370, 1980.

[32] S. Ladhani, O. S. Konana, S. Mwarumba, and M. C. English, "Bacteraemia due to Staphylococcus aureus," Archives of Disease in Childhood, vol. 89, no. 6, pp. 568-571, 2004.

[33] J. Rello, E. Quintana, V. Ausina, C. Puzo, A. Net, and G. Prats, "Risk factors for Staphylococcus aureus nosocomial pneumonia in critically ill patients," American Review of Respiratory Disease, vol. 142, no. 6, pp. 1320-1324, 1990.

[34] M. Pedersen, T. L. Benfield, P. Skinhoej, and A. G. Jensen, "Haematogenous Staphylococcus aureus meningitis: a 10-year nationwide study of 96 consecutive cases," BMC Infectious Diseases, vol. 6, article 49, 2006. 


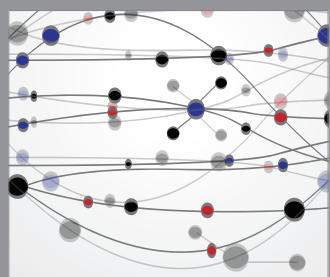

The Scientific World Journal
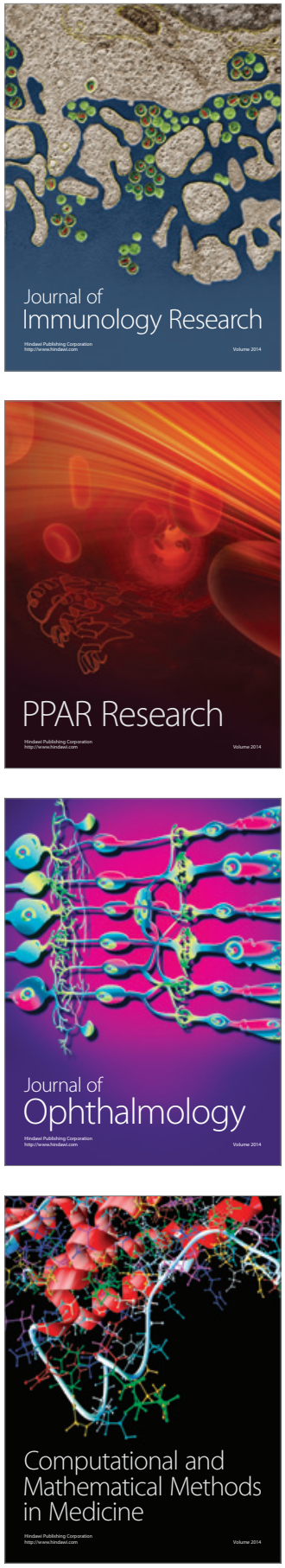

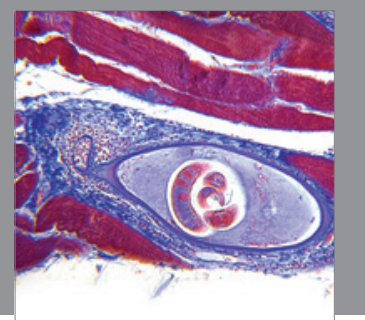

Gastroenterology

Research and Practice
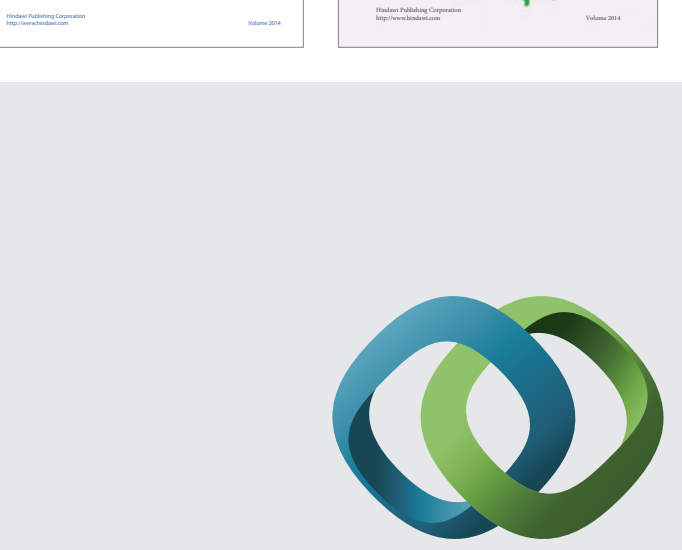

\section{Hindawi}

Submit your manuscripts at

http://www.hindawi.com
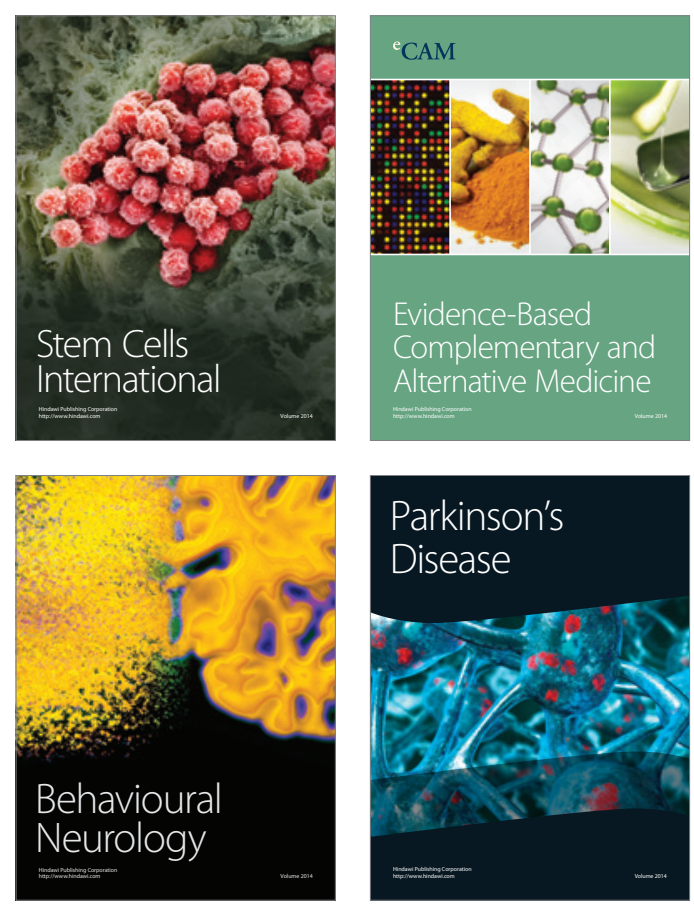

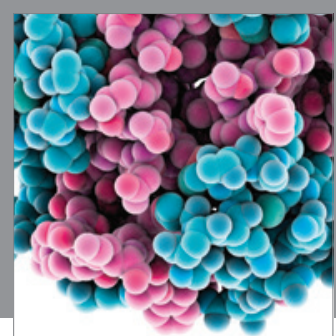

Journal of
Diabetes Research

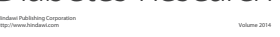

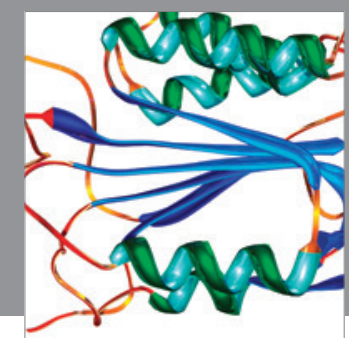

Disease Markers
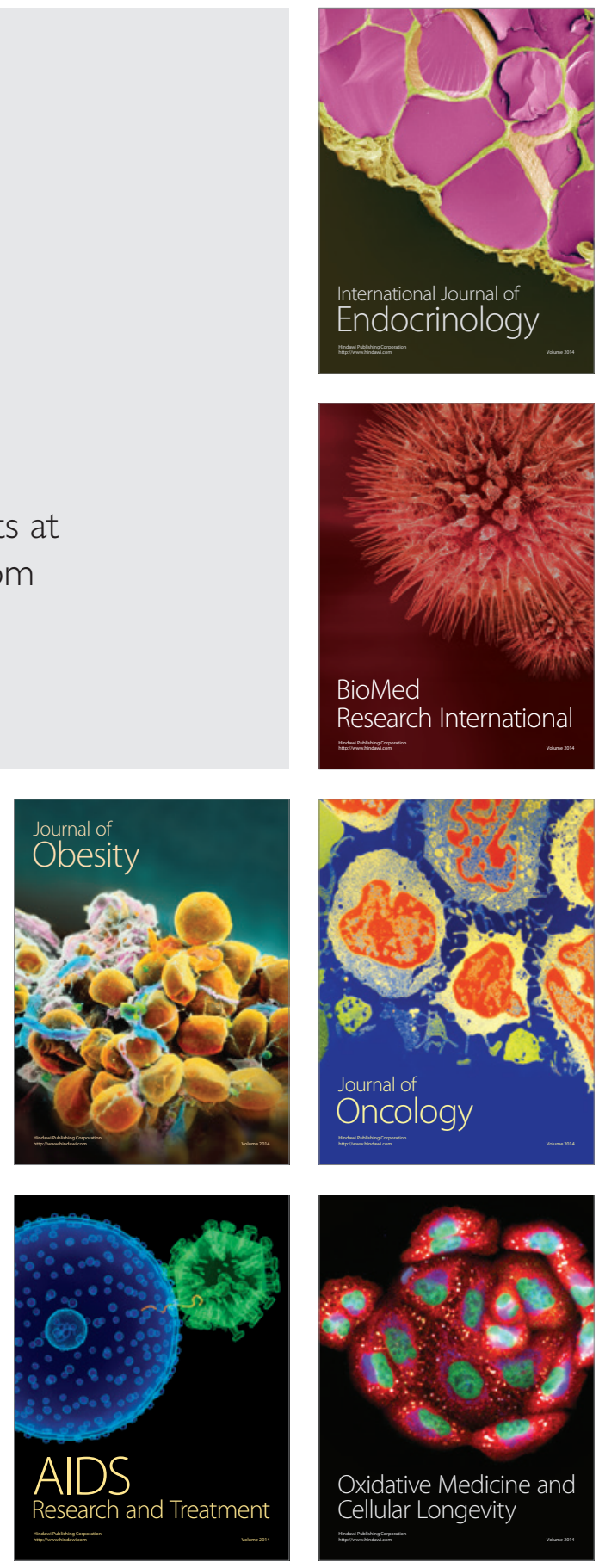